\title{
Electo protector de la melatonina y del tratamiento tópico con la mezcla eutéctica de lidocaínay prilocaína en un modelo de isquemia repertusión en el colgajo cutáneo microvascularizado en ratas
}

\section{Protective effect of melatonin and the lidocaine and prilocaine eutectic mixture in an ischemia reperfusion injury model in the microvascular cutaneous flap in rats}

\author{
Casado Sánchez, C. ${ }^{*}$, Aguilar Melero, P. **, González Menchén, A.***, Galache Collell, J.*, \\ San José Valiente, B. ${ }^{* \star *}$, Rioja Torrejón, L.*****, Padillo Ruiz J. ******
}

Resumen

Abstract

El síndrome de isquemia reperfusión es el conjunto de sucesos desarrollados desde la instauración de la isquemia en un tejido hasta su posterior reperfusión. Se trata de una condición limitante y, hasta la fecha, inevitable, en toda cirugía que implique una revascularización tisular. En un intento por buscar medidas terapéuticas frente al estrés oxidativo desarrollado durante este síndrome en los colgajos microvascularizados, se valoró la acción del antioxidante melatonina y de los anestésicos locales lidocaína y prilocaína en un modelo de isquemia reperfusión en el colgajo epigástrico microvascularizado en ratas. Tanto el indol como los fármacos vasoactivos poseen un efecto protector en el tratamiento del síndrome de isquemia reperfusión, desde un punto de vista bioquímico e histológico, destacando su acción sinérgica manifestada principalmente como un incremento en la neovascularización tisular.
Ischemia-reperfusion injury is a set of events developed since the introduction of ischemia in a tissue to subsequent reperfusion. It is a limiting condition and, to date, inevitable in any surgery involving tissue revascularization. In an attempt to find therapeutic measures against oxidative stress developed during this syndrome in microvascular flaps, we evaluated the antioxidant action of melatonin and local anesthetics lidocaine and prilocaine in a model of ischemia reperfusion in the microvascularized epigastric flap in rats. The indole and vasoactive drugs have a protective effect in the treatment of ischemia reperfusion injury, from both a biochemical and histological view, emphasizing their synergistic action mainly manifested as an increase in tissue neovascularization.

\author{
Palabras clave Síndrome de isquemia reperfusión, \\ Melatonina. EMLA. \\ Código numérico 1583-158336
}

\begin{tabular}{|ll|}
\hline Key words & $\begin{array}{l}\text { Ischemia reperfusion injury, } \\
\text { Melatonin, EMLA. }\end{array}$ \\
Numeral Code & $1583-158336$
\end{tabular}

\footnotetext{
* Médico Interno Residente del Servicio de Cirugía Plástica y Reparadora, Hospital Universitario Reina Sofía, Córdoba. España.

** Bióloga. Unidad de Investigación Hepática, Instituto Maimónides de Investigación Biomédica de Córdoba. España.

*** Especialista del Servicio de Anatomía Patológica, H.U. Reina Sofía, Córdoba. Esapaña.

**** Estadística, Actividad privada, Madrid. Esapaña.

***** Jefe de Servicio de Cirugía Plástica y Reparadora, H.U. Reina Sofía, Córdoba. España.

****** Jefe de Servicio de Cirugía General y Aparato Digestivo, H.U. Virgen del Rocío, Sevilla. España.
}

Trabajo galardonado con el Premio de Residentes de la Sociedad Española de Cirugía Plástica, Reparadora y Estética (SECPRE), Gerona (España), mayo del 2010; con la Beca de Investigación de la Fundación Docente de la Federación Iberolatinoamericana de Cirugía Plástica (FILACP) en Panamá (Panamá), junio del 2010, y con el Segundo Premio Internacional de Residentes de la FILACP en Medellín (Colombia), mayo del 2012. 


\section{Introducción}

La isquemia es una situación de inadecuado flujo sanguíneo en un área tisular específica que origina un deficiente aporte de oxígeno y nutrientes. Tras la reperfusión o restablecimiento del flujo sanguíneo, una serie de acontecimientos fisiopatológicos provocan un incremento del daño tisular (1). El conjunto de sucesos durante la isquemia y la reperfusión de un tejido se denomina síndrome de isquemia reperfusión (SIR).

El SIR, con sus complejas modificaciones moleculares y bioquímicas, supone una limitación fundamental en toda cirugía que implique revascularización tisular, como es la de los reimplantes y trasplantes tisulares, siendo una causa importante de disfunción de órganos trasplantados, provocando su fracaso y una mayor incidencia en el rechazo agudo o crónico del órgano (1-7).

La agresividad del SIR se basa en la producción de reactivos sintetizados en los tejidos sometidos a un período de isquemia, conocidos como radicales libres de oxígeno (RLO), caracterizados por poseer un número impar de electrones en las órbitas periféricas lo que les confiriere un estado reactivo y agresivo con los tejidos. Los RLO más abundantes y reactivos en los sistemas biológicos son el oxígeno molecular $\left(\mathrm{O}_{2}\right)$ y los productos derivados de su reducción tetravalente en agua, como el anión superóxido $\left(\mathrm{O}_{2}-\right)$, el peróxido de hidrógeno $\left(\mathrm{H}_{2} \mathrm{O}_{2}\right)$ y el radical hidroxilo (OH-).

Para contrarrestar la acción de los RLO, las células están dotadas de un potente armamento defensivo compuesto de mecanismos neutralizantes y formado por enzimas y compuestos moleculares y transportadores que evitan tanto la formación incontrolada y excesiva de RLO, como facilitan la neutralización, eliminación y bloqueo de su reactividad en cadena. Este sistema es conocido como defensa antioxidante.

De la falta de equilibrio entre los fenómenos pro-oxidantes y los mecanismos de defensa antioxidante en favor de los primeros, se deriva lo que ha venido en llamarse estrés oxidativo (EO), característico del SIR.

Entre los múltiples mecanismos antioxidantes, destaca el papel sistémico de la melatonina. Constituye la principal sustancia secretada por la glándula pineal, siendo su síntesis y su liberación estimuladas por la oscuridad e inhibidas por la luz. Entre sus múltiples funciones destacan su papel en la regulación biológica de los ritmos circadianos, el sueño, el humor, en la fisiopatología de la reproducción así como en el envejecimiento celular. Tiene capacidad inmunomoduladora, inhibe el crecimiento tumoral, influye en la fisiología de la retina, además de ser el antioxidante endógeno más potente conocido (8-13). En los últimos años se estudia experimentalmente su uso en diferentes procesos en los que median los RLO. A su carencia de toxicidad, se le asocia una gran facilidad para cruzar las barreras morfofisiológicas y entrar en los compartimentos subcelulares (14).
Estas propiedades la podrían convertir en un mecanismo útil en la prevención del SIR en los colgajos cutáneos.

Por otra parte, entre otras medidas preventivas y terapéuticas del SIR cabría valorar el papel relevante del tratamiento tópico con la mezcla eutéctica de lidocaína y prilocaína (EMLA ${ }^{\circledR}$ crema) en la prevención de la necrosis de los colgajos (15). Se trata de dos anestésicos locales con efectos bifásico sobre el músculo liso de los vasos sanguíneos; a concentraciones muy bajas producen vasoconstricción, mientras que a concentraciones habituales actúan como vasodilatadores (16). Su efecto sobre la isquemia tisular y las repercusiones que ésta genera, vendría caracterizado por su acción antagonista frente al vasoespasmo y la vasoconstricción.

En una estrategia terapéutica encaminada a controlar el SIR como mecanismo patogénico en los colgajos cutáneos microvascularizados cabría comparar ambas vías terapéuticas (bloqueo de radicales libres de oxígeno en el caso de la melatonina y vasodilatación en el caso de EMLA $^{\circledR}$ crema) y plantear si existe un efecto protector tisular sinérgico de ambas opciones.

Los objetivos de nuestro trabajo serán:

General: Analizar el papel del estrés oxidativo y la acción de la terapias antioxidante y vasodilatadora en un modelo experimental de isquemia reperfusión en los colgajos cutáneos microvascularizados.

Específicos:

- Evaluar la repercusión de la actividad de los radicales libres de oxígeno y de las enzimas antioxidantes en un modelo de isquemia-reperfusión en colgajos cutáneos microvascularizados.

- Determinar la inducción de apoptosis y de otros indicadores de sufrimiento tisular en un modelo de isquemia-reperfusión en colgajos cutáneos microvascularizados.

- Determinar el efecto de la melatonina como terapéutica antioxidante en la lesión de isquemia-reperfusión en los colgajos cutáneos microvascularizados.

- Determinar el efecto de la mezcla eutéctica de lidocaína y prilocaína como terapéutica en la lesión de isquemia-reperfusión en los colgajos cutáneos microvascularizados.

- Determinar el posible efecto sinérgico de la melatonina y de la mezcla eutéctica de lidocaína y prilocaína como terapéutica en la lesión de isquemia-reperfusión en colgajos cutáneos microvascularizados.

\section{Material y método}

El presente estudio se ha diseñado en base a un modelo experimental de isquemia reperfusión en el colgajo epigástrico en la rata Wistar. Todos los especímenes empleados fueron machos de peso comprendido entre los 160 y los 260 gr. Los animales tuvieron un período de acomodación de 7 días previos a su entrada en el protocolo experimental en los estabularios donde se mantu- 
vieron. En el postoperatorio, fueron acomodados de forma individual, tuvieron libre acceso a la comida y al agua y fueron expuestos a ciclos de 12 horas de luz alternados con 12 horas de oscuridad.

\section{Inducción del síndrome de isquemia reperfusión en el colgajo.}

La inducción del SIR en el colgajo cutáneo del animal seleccionado se ha realizado en base a investigaciones previas establecidas. Es un modelo fácilmente reproducible, sobre una anatomía constante, que cuenta con numerosas ventajas a la hora de valorar múltiples parámetros experimentales $(17,18)$.

Asistido por anestesia intraperitoneal (ketamina 75 $\mathrm{mg} / \mathrm{kg}$ y xilacina $8 \mathrm{mg} / \mathrm{kg}$ ), se disecó en cada animal un colgajo cutáneo de 7 x $4 \mathrm{~cm}$ sobre el hemiabdomen izquierdo. Este colgajo presenta un flujo axial basado en los vasos epigástricos inferiores, ramas directas de los vasos femorales, con origen localizado distalmente al ligamento inguinal y de dirección ventral. El colgajo fue elevado a modo de colgajo en isla (dejando su pedículo vascular como único vínculo con el animal).

Se procedió a la inducción de la isquemia en el colgajo aplicando un clamp microvascular simple en los vasos femorales de forma proximal a la salida del pedículo del colgajo y ligando los vasos femorales distalmente a él (Fig.1). A continuación se reposicionó el colgajo empleando sutura de seda 5/0 (Fig. 2).

En un período de 6 ó 12 horas más tarde, según el grupo, y asistido por una nueva anestesia, se procedió a realizar el desclampado del pedículo vascular mediante un mínimo abordaje en la línea de sutura inferior del colgajo. Se comprobó su adecuada permeabilidad, y se suturó de nuevo el colgajo en el territorio donante abdominal (Fig. 3-5).

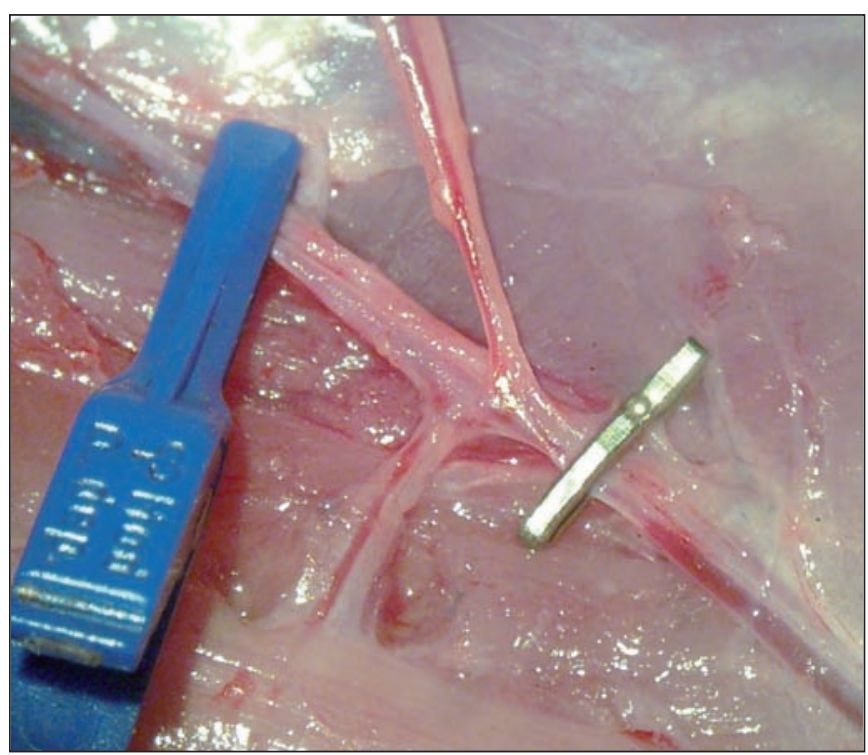

Fig. 1. Esquema del pedículo vascular. Vasos femorales ligados distalmente y clampados proximalmente al pedículo.

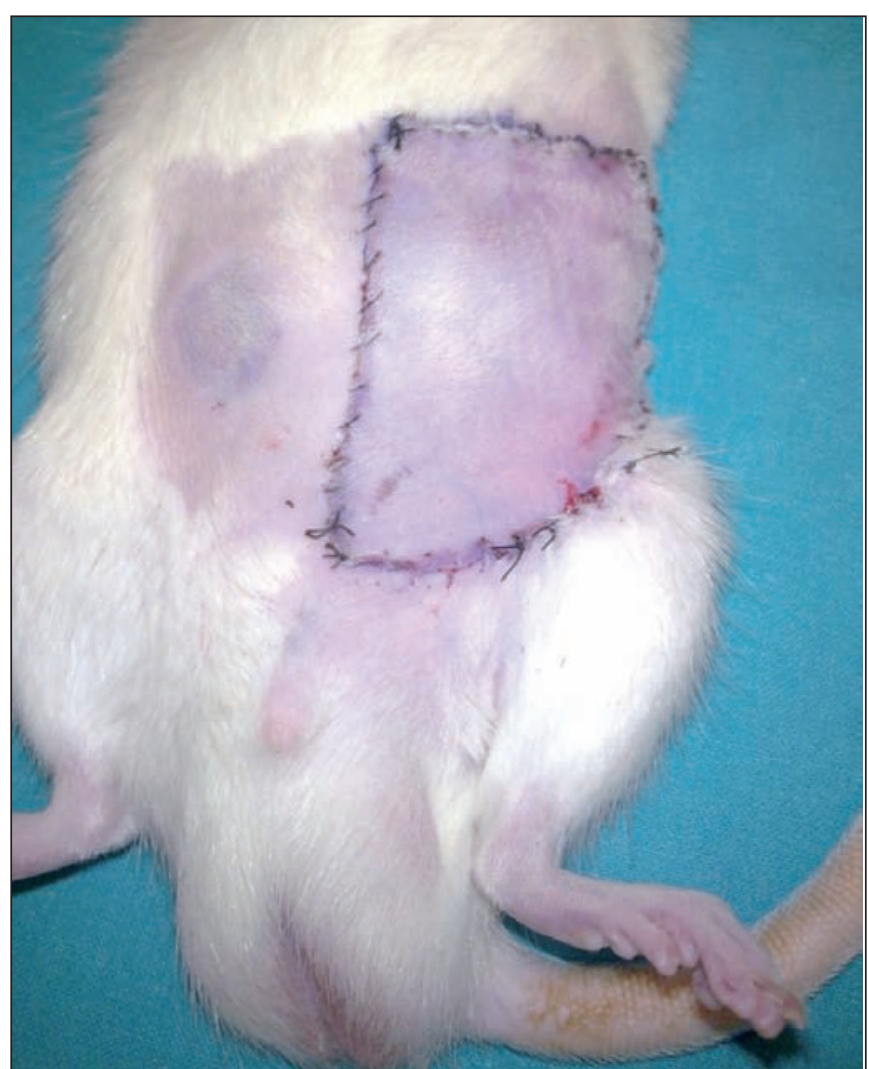

Fig. 2. Reposicionamiento del colgajo. Se mantiene el clamp vascular 6 ó $12 \mathrm{~h}$.

\section{Pautas de administración de melatonina y mezcla de lidocaína y prilocaína}

En los animales asignados a los grupos en los que se evaluarían los efectos de la melatonina (ver diseño del estudio), ésta se administró mediante una inyección por vía intraperitoneal en dosis de $4 \mathrm{mg} / \mathrm{Kg}, 1$ hora antes de la disección del colgajo, 30 minutos antes de la reperfusión, repitiéndose una dosis de forma diaria durante los 7 días transcurridos hasta la toma de muestras $(4 \mathrm{mg} / \mathrm{Kg}$ cada 24 horas) (Fig. 6).

En los animales asignados a los grupos en los se evaluarían los efectos de la aplicación de lidocaína y prilocaína (ver diseño del estudio), ésta se efectuó mediante la aplicación de $2 \mathrm{ml}$ de la crema sobre la superficie del colgajo tras la reperfusión del mismo, repitiéndose la aplicación de $2 \mathrm{ml}$ cada 8 horas de forma periódica durante los 7 días transcurridos hasta la toma de muestras (aplicaciones asistidas por anestesia mediante sevoflurano). Tras cada aplicación de EMLA ${ }^{\circledR}$ crema, el colgajo se cubría mediante un apósito Tegaderm ${ }^{\mathrm{TM}}$ film fijado con grapas cutáneas (Fig. 7).

\section{Sacrificio y toma de muestras}

Los animales fueron sacrificados en el momento de la toma de muestras, realizada a los 7 días del SIR. Bajo anestesia intraperitoneal empleando pentothal sódico $(50 \mathrm{mg} / \mathrm{kg})$, se practicó laparotomía media supra-infraumbilical con corte frío. Para una mejor exposición del diafragma se procedió a ampliar lateralmente la laparotomía (de esta manera se puede empujar caudalmente 

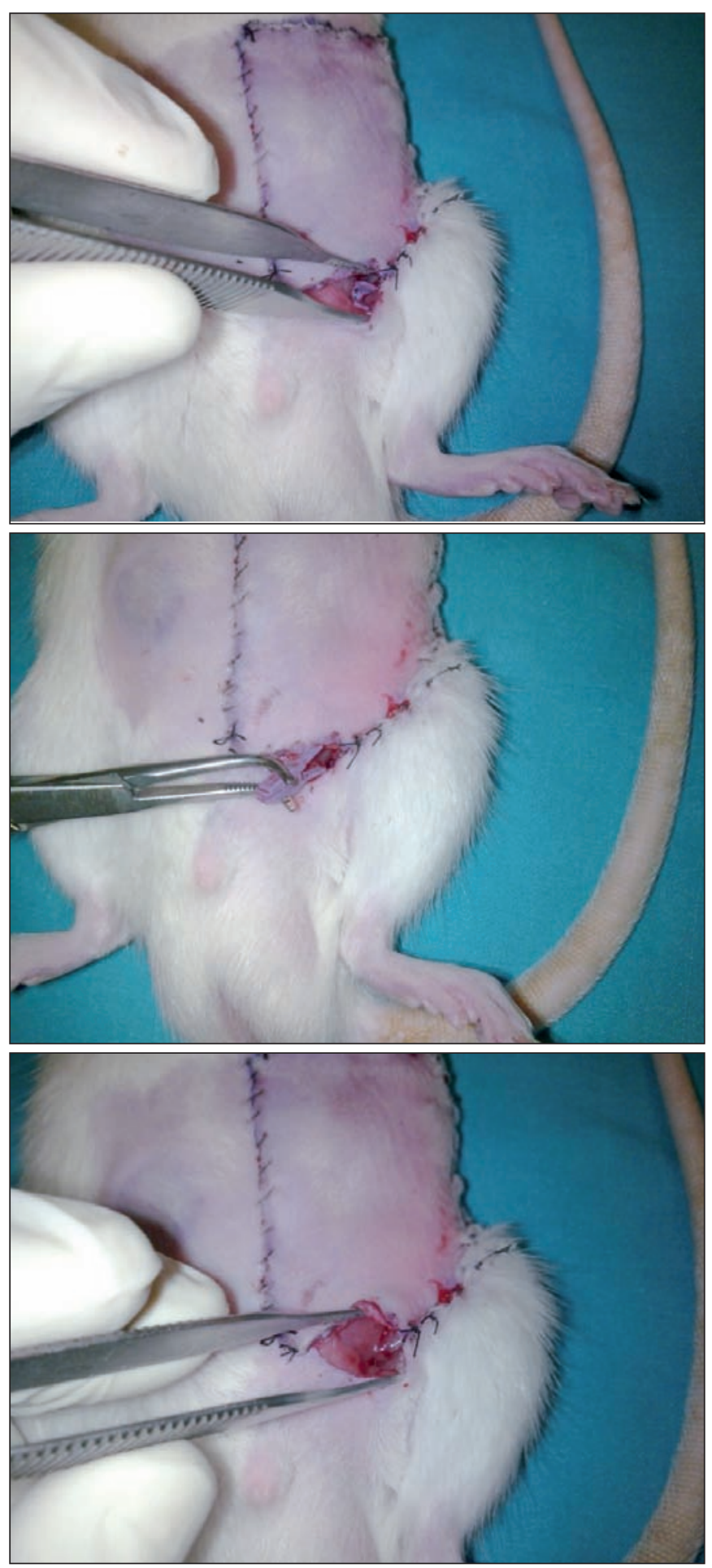

Fig. 3, 4, 5. Desclampado del pedículo y reperfusión del colgajo.

la cúpula hepática para poder acceder a la cavidad torácica). Se procedió seguidamente a la apertura del diafragma para la exanguinación del animal mediante punción cardíaca (Fig. 8).

La sangre fue almacenada en tubos Vacutainer $^{\circledR}$ $\mathrm{K}_{3}$ EDTA. Los tubos se dejaron 45 minutos a $4^{\circ} \mathrm{C}$ para ser posteriormente procesados (fueron centrifugados durante 5 minutos a 4500 revoluciones por minuto (rpm) y se transfirió el sobrenadante a 5 tubos Eppendorf que se congelaron a $-80^{\circ} \mathrm{C}$ para determinaciones analíticas posteriores).

Para la toma de muestras del colgajo, primero se rea-

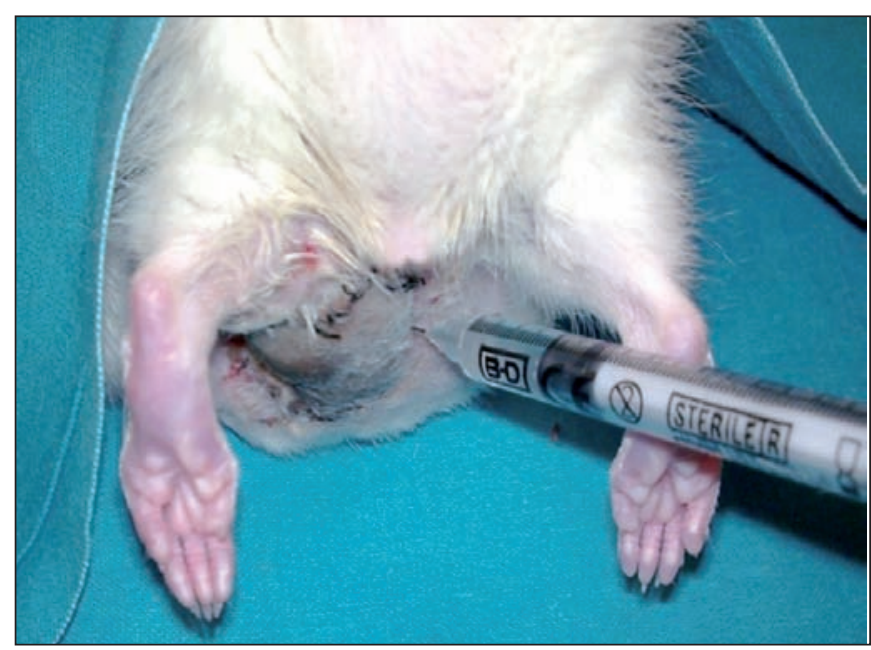

Fig. 6. Aplicación de $4 \mathrm{mg} / \mathrm{Kg}$ de melatonina i.p. en los animales de los gru-

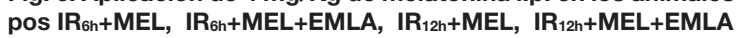

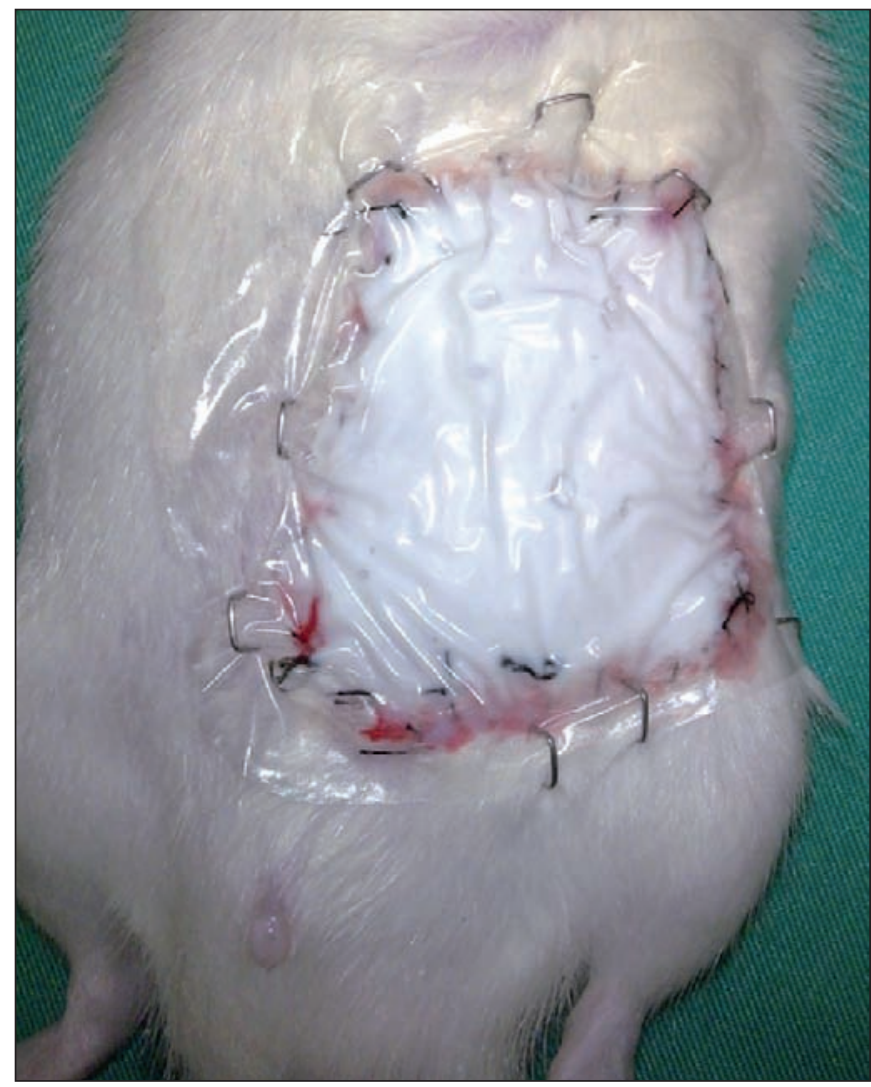

Fig. 7. Aplicación de $2 \mathrm{ml}$ de EMLA crema ${ }^{\circledR}$ en los animales de los grupos

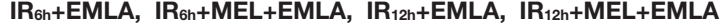

lizó examen macroscópico del colgajo, registrados mediante fotografía digital para posterior correlación. Se separaron fragmentos de $1 \times 4 \mathrm{~cm}$ que se almacenaron en criotubos para ser congelados inmediatamente en nitrógeno líquido (Fig. 9 y 10). Un fragmento se conservó en un bote de orina con $25 \mathrm{ml}$ de parafolmaldehido al $10 \%$, muestra que se utilizó para estudio anatomopatológico.

\section{Diseño del estudio}

Estudio experimental prospectivo randomizado triple ciego en el que se han empleado 63 ratas Wistar agrupados en 3 grupos experimentales: 


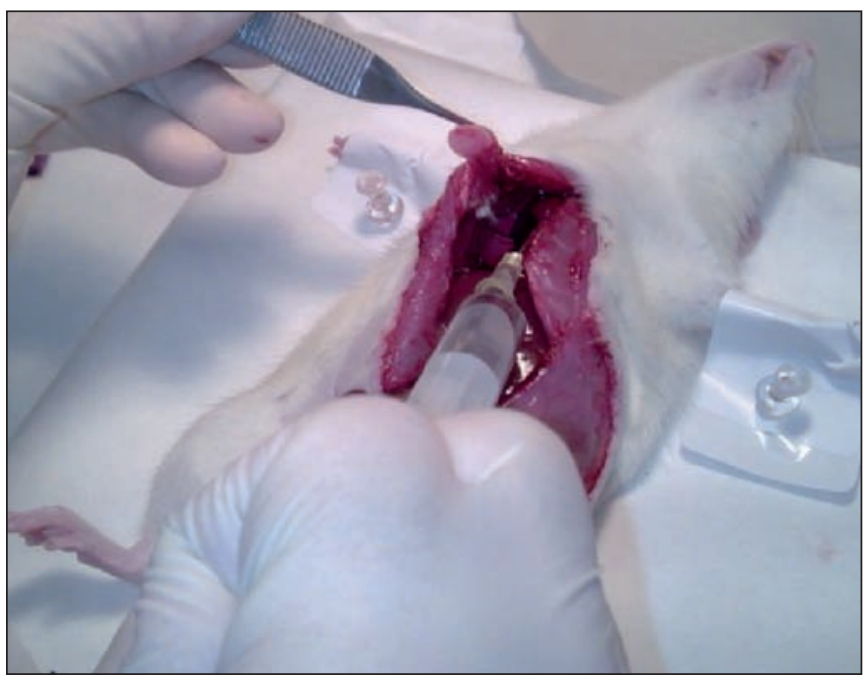

Fig. 8. Toma de muestras: extracción de sangre.
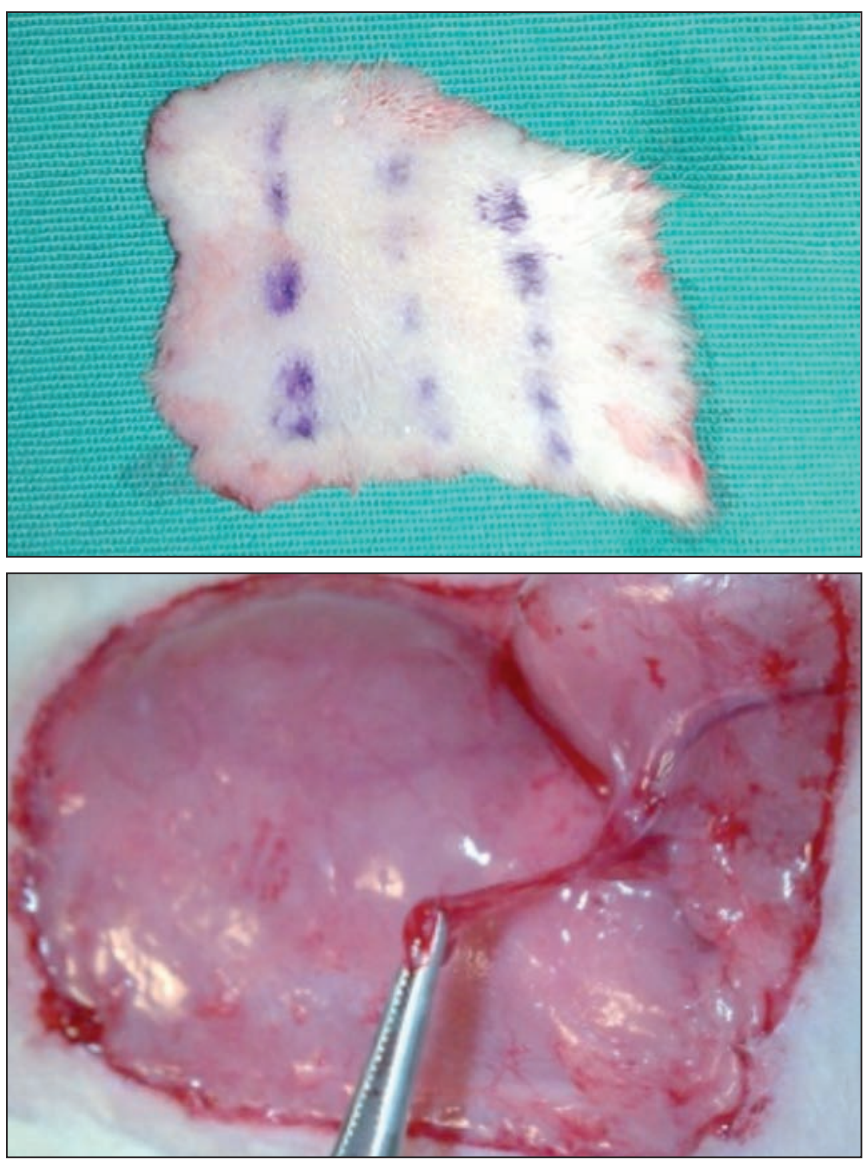

Fig. 9, 10. Toma de muestras: división en 4 fragmentos del colgajo.

- Grupo control (GC) formado por 7 animales en los que el colgajo disecado no fue sometido a isquemia (no se provocó un síndrome de isquemia reperfusión).

- Grupo 1 formado por 28 animales en los que se indujo un síndrome de isquemia reperfusión con una duración de la isquemia de 6 horas (6h).

- Grupo 2 formado por 28 animales en los que se indujo un síndrome de isquemia reperfusión con una duración de la isquemia de 12 horas (12h).
A su vez, los grupos 1 y 2 fueron divididos en 4 subgrupos de 7 animales cada uno de acuerdo al procedimiento al que fueran a someterse a los animales pertenecientes a cada uno de ellos. La descripción de los subgrupos es la siguiente:

- IR: animales a los que se sometió al SIR del colgajo sin la administración de ninguna sustancia química en estudio.

- IR + MEL: animales a los que se les sometió al SIR del colgajo y se les administró melatonina.

- IR + EMLA: animales a los que se les sometió al SIR del colgajo y se les administró lidocaína más prilocaína.

- IR + MEL + EMLA: animales a los que se les sometió al SIR del colgajo y se les administró melatonina y lidocaína más prilocaína.

\section{Parámetros de estudio}

- Parámetros bioquímicos de estrés oxidativo:

- Glutatión total (GSH total): suma del glutatión oxidado y del glutatión reducido.

- Relación glutatión reducido / glutatión oxidado (GSH / GSSG):

* Glutatión reducido (GSH, sustrato de la reacción que transforma el peróxido de hidrógeno en agua mediante la enzima glutatión peroxidasa, actuando así como un antioxidante).

* Glutatión oxidado (GSSG, resultante de la oxidación del glutatión).

- Superóxido dismutasa (SOD): enzima antioxidante.

- Sustancias que reaccionan con el ácido tiobarbitúrico (TBARS, siendo MDA o malondialdehido su principal exponente): productos derivados de la lipoperoxidación o metabolismo lipídico, cuya cuantificación resulta una medida del proceso de oxidación.

- Parámetros histológicos de afectación tisular:

Las lesiones titulares de evolución tórpida del colgajo, se clasifican de acuerdo con criterios de Anatomía Patológica (mismos criterios anatomopatológicos que en los seres humanos).

Se han valorado como criterios anatomopatológicos de daño tisular el edema dermoepidérmico, el infiltrado inflamatorio, la apoptosis y la neovascularización.

- Parámetros morfológicos (variaciones de masa corporal) y de supervivencia.

\section{Análisis estadístico}

Los parámetros definidos fueron analizados empleando el programa estadístico SPSS ${ }^{\circledR} 9.0$ (SPSS Inc.). La descripción de los datos cualitativos se realiza en forma de frecuencias absolutas y porcentajes o proporciones (\%), y los datos cuantitativos mediante media como medida de tendencia central y la desviación típica o standard (DS). 
En la comparación de datos cuantitativos entre dos grupos se empleó el test de la U-Mann Whitney. Los datos cualitativos se compararán mediante el test de la $\mathrm{Chi}^{2}$ ) o el test exacto de Fisher para frecuencia esperada menor o igual a 5. Todas las pruebas estadísticas y contrastes de hipótesis se han considerado bilaterales y como valores significativos, aquellos cuyo nivel de confianza es del 95\% (valores de p inferiores a 0,05 ).

\section{Resultados}

La supervivencia de los animales al final del estudio fue del $100 \%$. Respecto a las variaciones ponderales, se apreció un incremento en la masa de todos los individuos, excepto en aquellos del grupo control y del grupo sometido a un período de isquemia de 6 horas sin ningún tratamiento asociado.

\section{GRUPOS CON INDUCCIÓN DEL SIR SIN TRATAMIENTO}

\section{- Grupo con isquemia de 6 horas frente a grupo control: \\ - Resultados bioquímicos (Tablas I-IV)}

No se han apreciado diferencias significativas en el comportamiento de estas variables.

\section{- Resultados histológicos (Tabla V)}

Se apreció un edema dermoepidérmico en todos los animales sometidos al período de isquemia (100\%), no observándose signos de edema a nivel cutáneo en el GC $(\mathrm{p}=0,001)$. Cuando se analizó el infiltrado inflamatorio, el $43 \%$ de todos los animales sometidos a isquemia se vieron afectados, no observándose diferencias estadísticamente significativas en relación a este parámetro $(p=0,192)$ respecto al grupo control. La presencia de fenómenos de apoptosis en las células de los colgajos pertenecientes a los animales sometidos a 6 horas de isquemia fue significativamente mayor respecto al grupo control (IR6h $14,29 \pm 10,18$ vs GC $0 ; \mathrm{p}=0,001$ ). Por último, los fenómenos apreciados de neovascularización siguientes al SIR (28\%) no muestran diferencias significativas respecto al grupo control.

- Grupo con isquemia de 12 horas frente a grupo control:

- Resultados bioquímicos (Tablas I-IV)

$\mathrm{Al}$ valorar los parámetros antioxidantes, la GSH total se encontró significativamente elevada en los animales del grupo sometido a 12 horas de isquemia respecto a los del grupo control $(\mathrm{p}=0,002)$. En cambio, la relación de GSH/GSSG alcanzó valores más elevados en el grupo control $(\mathrm{p}=0,046)$. Las diferencias encontradas al comparar el resto de parámetros bioquímicos no alcanzaron la significancia estadística, pese a presentar alguno de ellos una tendencia similar, como es el caso de la SOD.

- Resultados histológicos (Tabla V)

La aplicación de isquemia de 12 horas al colgajo de- terminó la aparición de edema e infiltrado inflamatorio en todos los animales, a diferencia de los controles, en los que no se desarrollaron estos fenómenos histológicos $(\mathrm{p}=0,001)$. De forma similar, las células de los colgajos sometidos a isquemia durante 12 horas expresaron fenómenos de apoptosis en el $34,29 \%$ de los casos, no determinándose apoptosis en los controles $(\mathrm{p}=0,001)$. La aplicación de isquemia no propició la génesis de neovascularización en los colgajos, presentando un comportamiento similar a los casos del grupo control.

- Grupo con isquemia de 6 horas frente a isquemia de 12 horas

- Resultados bioquímicos (Tablas I-IV)

Tras la aplicación de una isquemia de 12 horas se observó un incremento del estado de oxidación con niveles de glutatión oxidado significativamente mayores $(\mathrm{p}=0,043)$, acompañado de niveles más elevados de TBARS (IR6h 2,46 $\mu \mathrm{M}$; IR12h 2,84 $\mu \mathrm{M}$ ) y un descenso de la actividad antioxidante de SOD, si bien las diferencias observadas en estas últimas no fueron estadísticamente significativas.

- Resultados histológicos (Tabla V)

El análisis histológico de los colgajos sometidos a isquemias de 6 y 12 horas mostró la existencia de edema dermoepidérmico en todos los animales de ambos modelos, mientras que el infiltrado inflamatorio (IR6h 43\%; IR12h $100 \%$; $\mathrm{p}=0,070)$ y la presencia de fenómenos de apoptosis (IR6h 14,29\%; IR12h 34,29\%; p=0,005) fueron más frecuentes tras 12 horas de isquemia.

\section{RESULTADOS TRAS LA ADMINISTRACIÓN DE MELATONINA}

- Administración de melatonina en isquemia de 6 horas

- Resultados bioquímicos (Tablas I-IV)

No se aprecian diferencias estadísticamente significativas en los parámetros bioquímicos.

- Resultados histológicos (Tabla V)

Las comparaciones de los parámetros histológicos valorados señalaron una mejoría mediante el tratamiento con melatonina, aunque no alcanzan diferencias significativas.

- Administración de melatonina en isquemia de 12 horas

- Resultados bioquímicos (Tablas I-IV)

En los animales a los que se les administró melatonina, se observó una mejoría de las cifras obtenidas con un incremento de los indicadores antioxidantes como el GSH total, la relación GSH/GSSG y la SOD y una disminución reseñable de los marcadores de estrés oxidativo, los TBARS, si bien las diferencias no fueron estadísticamente significativas.

- Resultados histológicos (Tabla V)

La administración de melatonina tras 12 horas de is- 
quemia se acompañó de un descenso del 30\% del infiltrado inflamatorio y una reducción significativa de los fenómenos de apoptosis tisular (IR12h 34,29\%; IR12h+MEL 6,43\%; $\mathrm{p}=0,001)$.

\section{RESULTADOS TRAS LA ADMINISTRACIÓN DE LIDOCAÍNA Y PRILOCAÍNA}

- Administración de lidocaína y prilocaína en isquemia de 6 horas

- Resultados bioquímicos (Tablas I-IV)

No se aprecian diferencias estadísticamente significativas en los parámetros bioquímicos entre los resultados de los animales sometidos a 6 horas de isquemia y los de los animales tratados con $\mathrm{EMLA}^{\circledR}$ crema.

\section{- Resultados histológicos (Tabla V)}

Respecto a los parámetros histológicos, se observa una reducción no significativa del infiltrado inflamatorio y de los fenómenos de apoptosis con la aplicación de EMLA $^{\circledR}$ crema. Se determinó la aparición de neovascularización en el colgajo de forma significativa, con un incremento superior al $70 \%$ respecto a la abstención terapéutica $(\mathrm{p}=0,021)$.

- Administración de lidocaína y prilocaína en isquemia de 12 horas

- Resultados bioquímicos (Tablas I-IV)

Respecto al GSH total, se encontró una mejoría significativa en la expresión de este antioxidante en los animales tratados con EMLA $^{\circledR}$ crema respecto a la abstención terapéutica del SIR con ese período de isquemia de 12 horas. De igual forma, se observó una relación GSH/GSSG significativamente mejor con el tratamiento con EMLA $^{\circledR}$ crema de ese SIR respecto a los animales que no recibieron ninguna alternativa terapéutica del mismo SIR. EMLA ${ }^{\circledR}$ crema provocó una reducción de la expresión de los productos de la lipoperoxidación, aunque estos efectos beneficiosos no alcancen la significancia estadística.

\section{- Resultados histológicos (Tabla V)}

La aplicación de EMLA crema ${ }^{\circledR}$ en el colgajo redujo el edema dermoepidérmico en un $29 \%$, así como el infiltrado inflamatorio en un $43 \%$. La apotposis apreciada en estos colgajos disminuyó de forma significativa ( $\operatorname{IR}_{12 \mathrm{~h}} 34,29 \%$; $\mathrm{IR}_{12 \mathrm{~h}}+\mathrm{EMLA} 7,147 \%$; $\left.\mathrm{p}=0,001\right)$, acompañándose de un incremento de la neovascularización de un $29 \%$.

\section{RESULTADOS TRAS LA ADMINISTRACIÓN CONJUNTA DE MELATONINA Y DE LIDOCAÍNA Y PRILOCAÍNA}

- Administración de melatonina y de lidocaína y prilocaína en isquemia de 6 horas

- Resultados bioquímicos (Tablas I-IV)

Como ocurrió en los apartados anteriores en los que se comparaba el modelo de IR $6 \mathrm{~h}$ con y sin tratamiento de dicho SIR, no se apreciaron diferencias estadísticamente significativas a la hora de valorar los parámetros bioquímicos de estrés oxidativo.

\section{- Resultados histológicos (Tabla V)}

Las comparaciones de los parámetros histológicos valorados no ofrecen diferencias estadísticamente significativas en relación a los cambios observados respecto a tratar o no el SIR inducido por un período de isquemia de 6 horas con MEL y EMLA ${ }^{\circledR}$ crema. Pese a no encontrar estas diferencias significativas, se aprecia un menor edema a nivel dermoepidérmico en los colgajos tratados mediante antioxidantes y vasodilatadores, acompañado de un infiltrado inflamatorio de menor intensidad y un incremento sustancial en la neovascularización del tejido afecto por el SIR y tratado con ambos fármacos; la agresividad histopatológica sería así menor.

- Administración de melatonina y de lidocaína y prilocaína en isquemia de $\mathbf{1 2}$ horas

- Resultados bioquímicos (Tablas I-IV)

Respecto a los parámetros bioquímicos evaluados, se encuentra una mejoría en todos los parámetros tras el tratamiento del SIR (inducido con 12 horas de isquemia) mediante la aplicación de las dos terapias en estudio de forma conjunta, melatonina y EMLA crema ${ }^{\circledR}$; se aprecia un aumento cercano a 5 veces mayor de los niveles de GSH total, un incremento en los valores de la relación GSH/GSSG, el aumento en la expresión del antioxidante SOD, así como un descenso en los niveles de TBARS.

- Resultados histológicos (Tabla V)

Las comparaciones de los parámetros histológicos valorados ofrecen diferencias estadísticamente significativas en las variables que hacen referencia a la presencia de neovascularización $\left(\mathrm{IR}_{12 \mathrm{~h}} 0 \%\right.$; $\mathrm{IR}_{12 \mathrm{~h}}+\mathrm{MEL}+\mathrm{EMLA}$ $100 \% ; \mathrm{p}=0,001)$ y fenómenos de apoptosis $\left(\mathrm{IR}_{12 \mathrm{~h}}\right.$ $34,29 \%$; IR ${ }_{12 \mathrm{~h}}+$ MEL+EMLA 9,29\%; $\left.\mathrm{p}=0,001\right)$. La disminución del infiltrado inflamatorio con los tratamientos disminuyó en un 43\%. Las diferencias en el edema epidérmico no son comparables puesto que en todos los casos se observaron signos de dicho edema. En general se aprecia una agresividad histopatológica menor en el grupo sometido a un SIR inducido mediante 12 horas de isquemia y tratado con MEL y EMLA ${ }^{\circledR}$ crema respecto al grupo con idéntico SIR, pero sin tratamiento asociado.

\section{ESTUDIO COMPARATIVO DE LAS TERAPÉUTICAS ADMINISTRADAS}

- IR 6 horas + MEL frente a IR 6 horas + EMLA frente a IR 6 horas + MEL + EMLA

- Resultados bioquímicos (Tablas I-IV)

En el modelo de SIR mediante 6 horas de isquemia, no se encuentran diferencias estadísticamente significativas entre las comparaciones de los parámetros bioquímicos obtenidos con cada una de las alternativas terapéuticas evaluadas. 


\section{- Resultados histológicos (Tabla V)}

Referidos a los parámetros histológicos, existieron diferencias estadísticamente significativas en la detección de fenómenos de apoptosis en los colgajos $(\mathrm{p}=0,002)$. Se observó este fenómeno con menor frecuencia en los que recibieron una única terapia como tratamiento del SIR inducido con 6 horas de isquemia frente a los que recibieron ambas (IR ${ }_{6 \mathrm{~h}}+\mathrm{MEL} 5,00 \%$; IR ${ }_{6 \mathrm{~h}}+\mathrm{EMLA} 5,00 \%$; $\left.\mathrm{IR}_{6 \mathrm{~h}}+\mathrm{MEL}+\mathrm{EMLA} 17,14 \% ; \mathrm{p}=0,002\right)$. Se observó una tendencia a presentar una neovascularización mayor empleando EMLA $^{\circledR}$ crema de forma individual o combinada con MEL respecto al empleo de forma individual de MEL. La magnitud de esta variación fue de hasta un $57 \%$ superior aplicando EMLA ${ }^{\circledR}$ crema respecto a la administración aislada de melatonina $(\mathrm{p}=0,070)$.

- IR 12 horas + MEL frente a IR 12 horas + EMLA frente a IR 12 horas + MEL + EMLA

- Resultados bioquímicos (Tablas I-IV)

La asociación de MEL y EMLA ${ }^{\circledR}$ crema ofreció valores muy elevados de GSH total respecto a los obtenidos mediante el empleo individual de las terapias $\left(\mathrm{IR}_{12 \mathrm{~h}}+\mathrm{MEL} 187,96 \mathrm{nmol} / \mathrm{mg} ; \mathrm{IR}_{12 \mathrm{~h}}+\mathrm{EMLA} 309,64\right.$ $\mathrm{nmol} / \mathrm{mg} ; \mathrm{IR}_{12 \mathrm{~h}}+\mathrm{MEL}+$ EMLA 543,64 $\pm 583,38$ $\mathrm{nmol} / \mathrm{mg}$ ). Al comparar los incrementos de SOD, se observó un incremento significativo al aplicar MEL, respecto al tratamiento con EMLA $^{\circledR}$ crema (IR ${ }_{12 \mathrm{~h}}+\mathrm{MEL} 322,93$ $\mathrm{U} / \mathrm{mg}$; IR $\left.{ }_{12 \mathrm{~h}}+\mathrm{EMLA} 57,96 \mathrm{U} / \mathrm{mg} ; \mathrm{p}=0,025\right)$. Analizando los TBARS, el descenso más relevante se observó al combinar las terapias ( $\left.\operatorname{IR}_{12 \mathrm{~h}}+\mathrm{MEL}+\mathrm{EMLA} 1,54 \mu \mathrm{M}\right)$.

- Resultados histológicos (Tabla V)

La tendencia de los fármacos a disminuir el infiltrado inflamatorio ofreció diferencias entre las alternativas planteadas $(p=0,057)$. Se apreció que el empleo de EMLA $^{\circledR}$ crema de forma aislada o combinada con MEL disminuía dicho infiltrado respecto al empleo aislado de MEL. Las diferencias descritas en apartados anteriores respecto al beneficio obtenido en términos de apoptosis, al tratar el SIR por isquemia de 12 horas con cual-

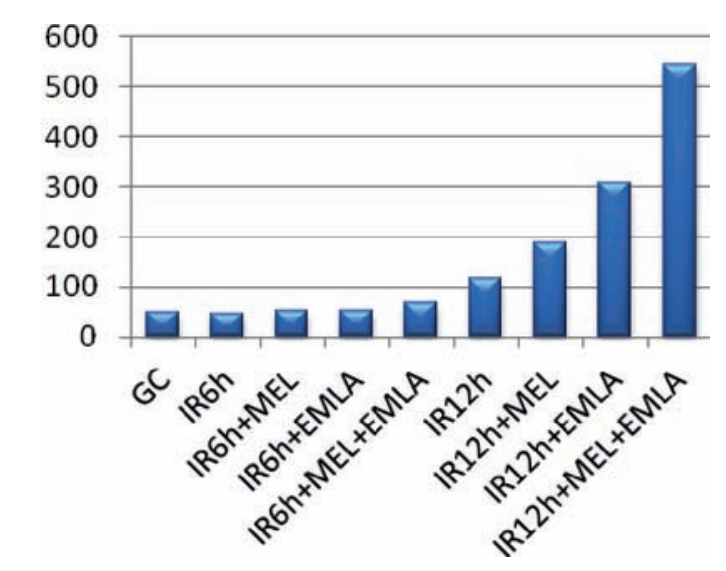

Tabla I. GSH Total (nmoles/mg)

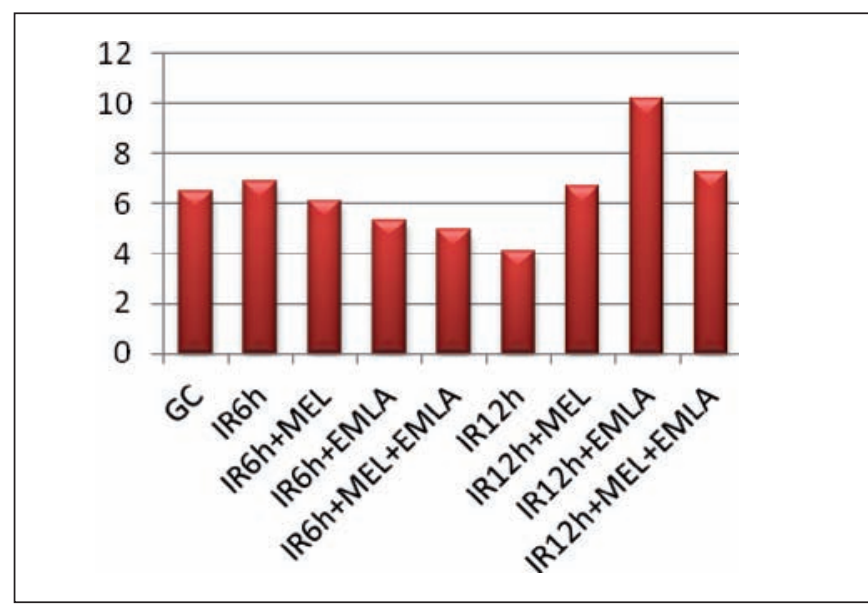

Tabla II. GSH/GSSG (nmoles/mg)

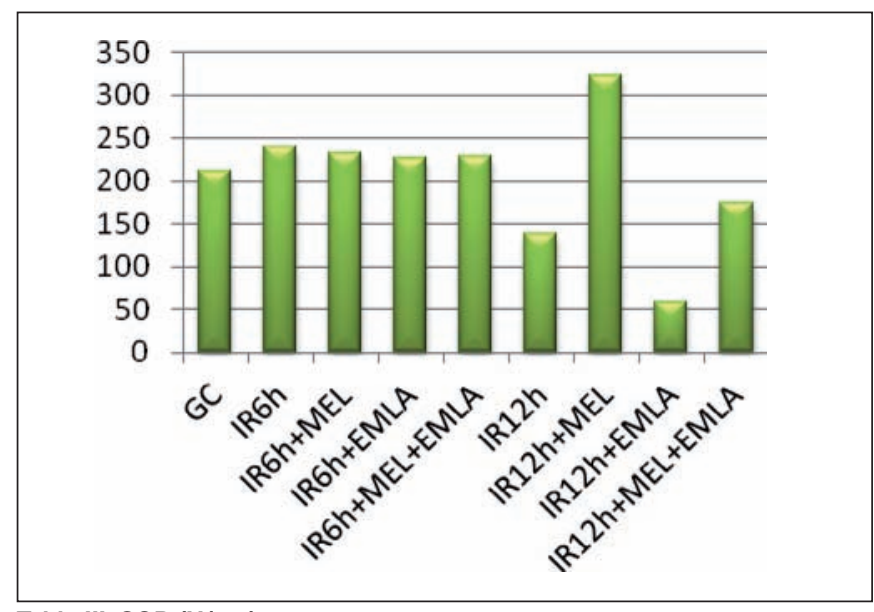

Tabla III. SOD (U/mg)

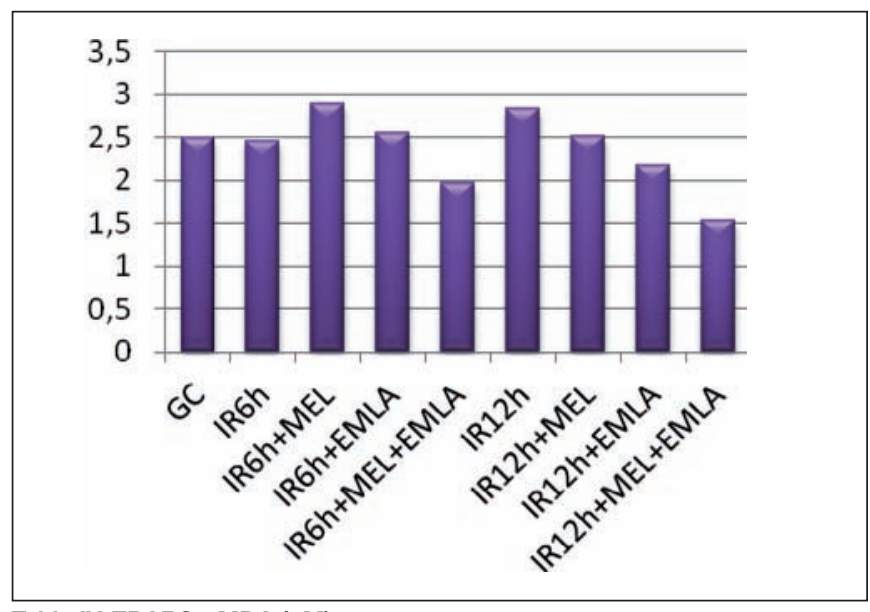

Tabla IV. TBARS - MDA $(\mu \mathrm{M})$

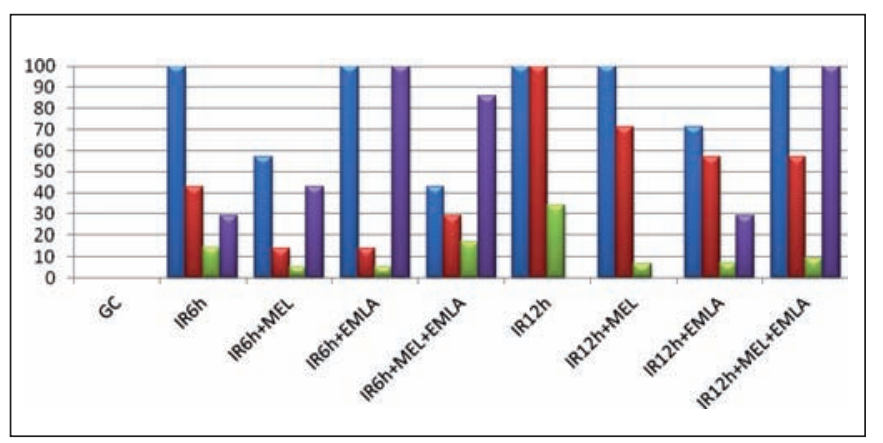

Tabla V. Marcadores anatomopatológicos. 
quiera de las alternativas propuestas respecto a la abstención terapéutica, no se aprecia al comparar las tres opciones, aunque se aprecia una mejoría al emplear MEL de forma aislada. Al valorar la neovascularización, se observaron diferencias estadísticamente significativas $(\mathrm{p}=0,000)$ al comparar este fenómeno en los animales que recibieron ambas terapias respecto a aquellos que recibieron una única terapia, $\operatorname{MEL}(\mathrm{p}=0,001) \mathrm{o}$ EMLA $^{\circledR}$ crema $(p=0,002)$, con presencia de neovascularización en todos los colgajos al combinarlos $\left(\mathrm{IR}_{12 \mathrm{~h}}+\mathrm{MEL}+\right.$ EMLA $\left.100 \%\right)$.

\section{Discusión}

El incremento en la supervivencia de los colgajos continúa siendo un objetivo prioritario en el desarrollo de la cirugía reconstructiva. Hasta la fecha, ningún fármaco empleado en la clínica diaria ha alcanzado todos los requisitos para convertirse en un mecanismo ideal para la consecución de este objetivo, debido a que los resultados de los ensayos clínicos son contradictorios o bien incluyen grupos heterogéneos dada la diversidad de las indicaciones, $\mathrm{u}$ otras posibles causas.

El modelo experimental de síndrome de isquemia reperfusión en el colgajo cutáneo microvascularizado en la rata Wistar presenta grandes ventajas al tener una gran similitud histológica con el humano y de ser de fácil reproducción y ejecución. Respecto al tiempo de isquemia, la mayoría de los autores seleccionan períodos de 6 a 12 horas para poder apreciar con facilidad efectos lesivos a nivel bioquímico y tisular.

Respecto a la inducción de la isquemia, el método preferido por diferentes autores consiste en la disección completa del colgajo y su pedículo vascular, a modo de colgajo en isla verdadero, incluyendo en esta disección a los vasos femorales con ligadura de éstos distal al pedículo epigástrico y aplicación de un clamp microvascular en los vasos femorales, proximal a los vasos epigástricos, durante el período de tiempo seleccionado. Se decidió utilizar este procedimiento descrito para la inducción de la isquemia, no seleccionando los vasos epigástricos para la inducción de la isquemia (procedimiento más rápido y técnicamente más sencillo, pero con vasos más frágiles susceptibles de sufrir lesión por presión mantenida de largo tiempo) y realizando la ligadura de los vasos femorales distal a la salida del pedículo epigástrico para evitar la reperfusión mediante flujo retrógrado.

Respecto al diseño de los grupos de estudio, éstos se definieron primero según un doble criterio: la inducción o no del síndrome de isquemia reperfusión y la duración del período de isquemia. No existen en la literatura modelos similares y se consideró interesante evaluar los parámetros bioquímicos e histológicos con ambos períodos de isquemia, de modo que se puedan evaluar y comparar la efectividad o no de las terapias en dichas circunstancias. El segundo criterio para subdividir a los animales en los grupos de estudio se basó en la aplicación de las medidas terapéuticas.

La melatonina, indolamina producida por la glándula pineal, es materia de múltiples estudios en los últimos años. Sus acciones se han relacionado con los ritmos circadianos, función inmune, sueño, fisiología de la retina y funciones endocrinas en general. Se ha descubierto que es un eficaz antioxidante y neutralizante (scavenger) de radicales libres. Estas propiedades, junto a sus características farmacocinéticas, hicieron que fuese objeto de estudio. Cruza con facilidad las barreras morfofisiológicas y penetra en los compartimentos subcelulares. Este efecto como tamponador directo de los radicales libres es complementario a su capacidad de activar otras enzimas antioxidantes. Cabe destacar los trabajos de Gurlek (19) donde se describe el efecto protector de la melatonina en un modelo de síndrome de isquemia reperfusión en el colgajo cutáneo microvascularizado en la rata Wistar. En su trabajo señala un efecto protector traducido en una reducción en los niveles de radicales libres y un incremento de los mecanismos intrínsecos de defensa antioxidante, con el beneficio final sobre la supervivencia tisular del colgajo.

En relación a la otra sustancia evaluada, cabría mencionar que hasta la presente publicación no existen estudios que hayan evaluado el efecto protector de la mezcla eutéctica de lidocaína y prilocaína sobre los colgajos cutáneos microvascularizados de flujo axial sometidos de forma experimental al síndrome de isquemia reperfusión. Sus posibles efectos terapéuticos se basan en sus propiedades vasoactivas: provocan una respuesta bifásica vascular que consiste en una vasoconstricción inicial máxima a los 90 minutos de la aplicación y una vasodilatación tardía en aplicaciones más largas de 3 horas. En estos cambios vasculares residiría su efecto beneficioso: la vasoconstricción inicial seguida de la vasodilatación sometería al colgajo a un fenómeno conocido como "preacondicionamiento isquémico", de naturaleza farmacológica. La aplicación tópica de este fármaco le confiere una serie de ventajas respecto a otros medicamentos en el tratamiento y prevención del síndrome de isquemia reperfusión. Podría alcanzar niveles terapéuticos adecuados en el tejido diana sin una distribución sistémica significativa y con un amplio margen de seguridad. Los referentes sobre los que se basa este aspecto de la investigación, son los trabajos de Karaçal (15). Los investigadores evaluaron los efectos de la mezcla eutéctica de lidocaína y prilocaína sobre la supervivencia de un colgajo cutáneo randomizado en la rata Wistar, señalando un incremento estadísticamente significativo en la supervivencia tisular de los colgajos tratados mediante la mezcla respecto al grupo control, sin hacer referencia en este estudio a parámetros bioquímicos o histológicos de valoración microscópica. Dado que las dimensiones del colgajo axial del presente estudio $\left(28 \mathrm{~cm}^{2}\right)$ son similares al colgajo empleado por Karaçal $\left(30 \mathrm{~cm}^{2}\right)$ y ante la au- 
sencia de otras investigaciones similares de referencia, se decidió la misma pauta empleada por este autor, disminuyendo la periodicidad de aplicación a 8 horas.

A la hora de valorar los resultados obtenidos y respecto a las variables bioquímicas, realizando una comparación general y antes de entrar en detalles particulares de los parámetros analizados, podemos decir que prácticamente no se encuentran diferencias entre los resultados obtenidos en el grupo control no sometido al síndrome de isquemia reperfusión y el grupo en el que se provocó un síndrome de isquemia reperfusión con un tiempo de isquemia de 6 horas. Sin embargo, sí se han encontrado importantes diferencias en el comportamiento de los parámetros bioquímicos cuando se realizaron las comparaciones entre dicho grupo control y el grupo experimental sometido a un síndrome de isquemia reperfusión con 12 horas de isquemia. Tras estas 12 horas de isquemia se afectan todos los parámetros, destacando el importante descenso de la relación GSH/GSSG, la disminución de la SOD y el aumento de la lipoperoxidación traducido como un incremento de los TBARS. Comparando globalmente los dos grupos de isquemia diferentes, se aprecia un nivel de estrés oxidativo mayor en el grupo de 12 horas de isquemia respecto a los observados tras 6 horas de isquemia. Esto nos llevaría a proponer un modelo de isquemia reperfusión basado en 12 horas de isquemia, ya que expresa de forma más evidente las alteraciones de los marcadores de estrés oxidativo.

El glutatión es considerado como uno de los antioxidantes más importantes, cuyos niveles disminuyen tras la isquemia reperfusión en los colgajos cutáneos en los animales. La inhibición de su producción endógena durante el SIR provocaría un incremento del daño tisular, y su administración antes de la misma lo disminuiría.

El valor más significativo para valorar la actividad de glutatión es la relación que existe entre el glutatión reducido y el glutatión oxidado (GSH/GSSG), ya que ante un estrés oxidativo relevante y según lo referido anteriormente, se oxidaría GSH, incrementándose los niveles de GSSG. Así pues, los niveles disminuidos de la relación GSH/GSSG son indicativos de una situación de estrés oxidativo importante. En nuestro estudio, así ocurre en el grupo de animales sometidos a 12 horas de isquemia en el que los niveles de GSH/GSSG fueron inferiores a los observados tras 6 horas de isquemia.

La superóxido dismutasa (SOD), sistema enzimático encargado de catalizar la reducción del radical superóxido en peróxido de hidrógeno, presenta valores inferiores tras períodos de isquemia de 12 horas respecto a los observados tras isquemia de 6 horas, en relación al mayor consumo de esta enzima en estados de estrés oxidativo más relevantes.

El malondialdehido (MDA), producto de la lipoperoxidación lipídica y principal exponente de las sustancias que reaccionan con el ácido tiobarbitúrico (TBARS, sustancias detectadas en el análisis bioquímico), se eleva de forma más relevante mediante perío- dos de isquemia de 12 horas respecto a las cifras observadas en el grupo sometido a un síndrome de isquemia reperfusión mediante un período de isquemia de 6 horas. Esto es indicativo de un estado de estrés oxidativo más avanzado mediante isquemia de 12 horas.

Respecto a los parámetros histológicos, de todos los resultados obtenidos merece la pena destacar varios aspectos. De forma genérica, señalar que tras 6 horas de isquemia se aprecia un edema dermoepidérmico y una apoptosis celular significativamente más acusados respecto al grupo control. Al incrementar esta isquemia a un período de 12 horas, además de aumentar la apoptosis celular respecto al grupo con 6 horas de isquemia (se aprecia incluso más del doble de afectación celular en el síndrome de isquemia reperfusión inducido tras 12 horas de isquemia, respecto al provocado tras inducciones con 6 horas), el infiltrado inflamatorio que se objetiva aumenta hasta afectar a todos los animales.

Períodos de isquemia de 12 horas son capaces de producir más diferencias estadísticamente significativas respecto al grupo control (3 parámetros de los 4 evaluados), de lo que lo harían tiempos de isquemia de 6 horas (solo se aprecian diferencias estadísticamente significativas en los parámetros de apoptosis y edema dermoepidérmico). Estos resultados apoyarían las tesis planteadas en otros estudios, que sugerían la necesidad de tiempos mayores de 6 horas para vislumbrar afectación de carácter macroscópico debido a la inducción de un síndrome de isquemia reperfusión (presencia de hasta un $78 \%$ de afectación macroscópica con isquemia de hasta 12 horas).

Al comparar los resultados obtenidos dentro del grupo sometido a 6 horas de isquemia para la inducción del SIR, se debe destacar que no existieron diferencias estadísticamente significativas en los parámetros bioquímicos evaluados entre los diferentes grupos de tratamiento. Esto es coherente con lo referido anteriormente $\mathrm{y}$ orienta hacia un comportamiento similar de esos parámetros en los individuos del grupo sometidos a 6 horas de isquemia y tratados con cualquiera de las alternativas. Se objetiva una similitud de los parámetros analizados que hacen referencia al glutatión (GSH total y GSH/GSSG), a la superóxido dismutasa y al malondialdehido (TBARS). Esta ausencia de diferencias significativas para los criterios bioquímicos evaluados dentro de ese período de isquemia, no significa que no se encontrasen diferencias para los criterios histológicos. El tratamiento individual con melatonina (respecto a la abstención terapéutica) disminuye la agresividad en el daño tisular, observándose en general un menor edema dermoepidérmico (con una disminución de un $43 \%$ respecto a la abstención terapéutica), un menor infiltrado inflamatorio (disminuyendo éste en un 29\%), una disminución de los fenómenos de apoptosis (disminuyendo en un $9 \%$ ) y un incremento en la neovascularización en un $14 \%$. Es interesante esta tendencia a disminuir la morbilidad histológica en estos períodos de isquemia (de 6 
horas) a pesar de no ser estadísticamente significativo. Respecto al empleo de la mezcla eutéctica de lidocaína y prilocaína, su aplicación tras 6 horas de isquemia mejora de forma significativa la neovascularización observada respecto a la abstención terapéutica (incrementando este fenómeno en un 71\%). Al igual que la melatonina, disminuye el infiltrado inflamatorio (en un 29\%) y los fenómenos de apoptosis (un 9\%) respecto a la abstención terapéutica. La combinación de ambas terapias no parece aportar una mejoría significativa en términos histológicos, en comparación al beneficio que implicarían de forma individual.

A diferencia de lo observado tras 6 horas de isquemia, al comparar los resultados obtenidos dentro del grupo sometido a 12 horas de isquemia para la inducción del síndrome de isquemia reperfusión, sí se observaron diferencias estadísticamente significativas en función de las pautas de tratamiento empleadas.

La melatonina posee efectos beneficiosos en el tratamiento del daño debido al estrés oxidativo, reflejado tanto a nivel bioquímico como histológico. A nivel bioquímico el tratamiento con melatonina actúa como mecanismo defensivo frente al estrés oxidativo a través de su acción sobre el glutatión (produciendo un incremento de sus nivele, ya que la enzima limitante en la síntesis de la GSH, la gamma-glutamilcisteína sintetasa, se encuentra elevada tras la administración de melatonina, o bien la melatonina produce un incremento directo en los niveles de GSH) y sobre el malondialdehido (la melatonina eliminaría directamente los radicales libres de oxígeno e incrementaría la actividad antioxidantes para reducir los niveles de MDA). El tratamiento con este potente antioxidante produjo beneficio a todos los niveles bioquímicos explorados: incrementa los niveles de GSH total con el beneficio de incrementar de la relación GSH/GSSG (esto apoya la tesis de un nivel de estrés oxidativo más controlado con el empleo de este neutralizante (scavenger), aumenta los niveles de la SOD y produce una regulación en los niveles de TBARS, indicando un descenso en la lipoperoxidación. Estos datos bioquímicos son paralelos a los hallazgos histológicos, pudiéndose afirmar que la melatonina mejora significativamente la presencia de fenómenos de apoptosis frente la abstención terapéutica (un descenso estadísticamente significativo cercano a un $28 \%$ ) y disminuye en un $30 \%$ el infiltrado inflamatorio.

Valorando la mezcla eutéctica de lidocaína y prilocaína en el tratamiento del síndrome de isquemia reperfusión inducido mediante un período de isquemia de 12 horas, cabe decir que los anestésicos poseen un efecto protector frente al daño generado por este síndrome tanto desde el punto de vista bioquímico como anatomopatológico.

Desde el punto de vista bioquímico, EMLA ${ }^{\circledR}$ crema aumenta, al igual que la melatonina, los niveles de GSH total, y posibilita un incremento en la relación GSH/GSSG de forma significativa. No se ha encontrado una relación entre el efecto beneficioso de la mezcla de anestésicos y su posible relación con el sistema enzimático de la SOD; incluso se ha detectado un incremento significativo de los niveles de SOD mediante la administración individual de melatonina respecto a los niveles obtenidos al utilizar de forma aislada la mezcla de anestésicos, al comparar sus efectos por separado en el tratamiento del síndrome de isquemia reperfusión con isquemia de 12 horas. Su aplicación se relaciona con un descenso en los niveles de TBARS, es decir, una disminución de la lipoperoxidación. Un estudio reciente (20), indicó el impacto de la lidocaína en la supervivencia de los colgajos microvascularizados experimentales tras un síndrome de isquemia reperfusión, presentando una disminución de los niveles de malondialdehído en los animales tratados con lidocaína. En su modelo experimental, la acción antioxidante de la lidocaína se centraría en la inhibición de la lipoperoxidación, siendo nuestros hallazgos acordes a este trabajo.

En términos histológicos, el empleo de EMLA $^{\circledR}$ crema disminuye la agresividad de forma general del síndrome de isquemia reperfusión en los tejidos afectos; desciende los valores del edema dermoepidérmico en un $29 \%$, del infiltrado neutrofílico en un $43 \%$ y disminuye significativamente los fenómenos apoptóticos respecto a la abstención terapéutica (disminuye la apoptosis en un 27\%, valor discretamente inferior al alcanzado por la melatonina). Por otra parte, aumenta la neovascularización en un $29 \%$. Cabe destacar que en los casos de tratamiento exclusivo con la mezcla de anestésicos, esta opción terapéutica es más efectiva en inducir mayor neovascularización en casos de síndrome de isquemia reperfusión inducidos con tiempos de isquemia menores (6 horas frente a 12 horas de isquemia).

Respecto al empleo combinado de ambas terapias, podemos afirmar que mejora las cifras de GSH total y de GSH/GSSG respecto a la abstención terapéutica y que la asociación de la mezcla de anestésicos y melatonina apuntan a una mejoría respecto al uso individual de ambos fármacos al referirnos al incremento de GSH total. También es relevante el descenso importante en los niveles de TBARS al asociar ambos, mayor que el obtenido al utilizarlos de forma individual. Desde el punto de vista anatomopatológico, como era esperable dado su comportamiento por separado, la asociación de ambos fármacos disminuye significativamente la aparición de fenómenos de apoptosis, y según lo expuesto anteriormente, esta reducción se produce a expensas de ambos medicamentos, fundamentalmente de la melatonina. Además, la asociación de la aplicación local de la mezcla de anestésicos junto a la administración sistémica de melatonina, mejora los resultados que la aplicación individual de melatonina tiene sobre el infiltrado inflamatorio (disminución de un $43 \%$ respecto la abstención terapéutica). Respecto a la neovascularización, importante factor en la evolución satisfactoria de los colgajos microquirúrgicos 
(principalmente en las áreas más periféricas), son destacables las diferencias significativas en relación a la combinación de ambas alternativas frente a su empleo por separado o la abstención terapéutica, mejorando e incrementando así la neovascularización al emplear de forma conjunta ambos fármacos. Al combinar ambas opciones terapéuticas, se consigue incrementar en un $100 \%$ la neovascularización objetivada en los colgajos microquirúrgicos sometidos a un período de isquemia de 12 horas.

En este sentido, y considerando nuestras condiciones experimentales, la combinación de ambos tratamientos parece una opción interesante en la prevención del síndrome de isquemia reperfusión.

\section{Conclusiones}

Los modelos de isquemia reperfusión en colgajos microvascularizados en ratas, basados en la inducción del SIR mediante períodos de isquemia de 12 horas, generan un incremento del estrés oxidativo con mayor intensidad que en los modelos basados en 6 horas. Tanto el empleo de melatonina como de EMLA ${ }^{\circledR}$ crema mejoran los marcadores de daño tisular a nivel bioquímico e histológico, pero además el empleo de ambos fármacos de forma combinada genera un efecto protector sinérgico, manifestado principalmente en la potenciación del desarrollo de la neovascularización en un 100\% de los colgajos.

\section{Conflicto de intereses}

El autor certifica que no tiene ninguna vinculación o compromiso con alguna organización o entidad con interés financiero directo o indirecto en el tema de trabajo.

\section{Dirección del autor}

Dr. César Casado Sánchez

Servicio de Cirugía Plástica y Quemados.

Hospital Universitario La Paz.

Paseo de la Castellana 261.

28046, Madrid.

e-mail: doctorcasado@gmail.com

\section{Bibliografía}

1. Cetin C, Kose AA, Aral E et al. Protective effect of fucoidin (a neutrophil rolling inhibitor) on ischemia reperfusion injury: experimental study in rat epigastric island flaps. Ann Plast Surg. 2001; 47: 540.

2. Rand-Luby L, Pommier RF, Williams ST et al. Improved outcome of surgical flaps treated with topical dimethylsulfoxide. Ann Surg. 1996; 224:583. Discussion 89.

3. Kuo YR, Wang FS, Jeng SF et al. Nitrosoglutathione promotes flap survival via suppression of reperfusion injury-induced superoxide and inducible nitric oxide synthase induction. J Trauma. 2004; 57: 1025.
4. Whitson BA, Nath DS, Johnson AC et al. Risk factors for primary graft dysfunction after lung transplantation. J Thorac Cardiovasc Surg. 2006; 131: 73.

5. Kupiec-Weglinski JW, Busuttil RW. Ischemia and reperfusion injury in liver transplantation. Transplant Proc. 2005; 37: 1653.

6. Burns AT, Davies DR, McLaren AJ et al. Apoptosis in ischemia/ reperfusion injury of human renal allografts. Transplantation. 1998; 66: 872.

7. Beyersdorf F. Myocardial and endothelial protection for heart transplantation in the new millenium: lessons learned and future directions. J Heart Lung Transplant. 2004; 23: 657.

8. Montilla P, Cruz A, Padillo FJ et al. Melatonin versus vitamin $\mathrm{E}$ as protective treatment against oxidative stress alter extra-hepatic bile Duch ligation in rats.J Pineal Res. 2001; 31(2): 138.

9. Rodríguez Reynoso S, Leal C, Portilla E et al. Effect of exogenous melatonin on hepatic energetic status during ischemia reperfusion: posible role of tumor necrosis factor-alpha and nitric oxide. J Surg Res. 2001; 100(2): 141.

10. Okatani Y, Wakatsuki A, Shinohara K et al. Melatonin protects against oxidative mitochondrial damage induced in rat placenta by ischemia and reperfusion. J Pineal Res. 2001; 31(2): 173 .

11. Cuzzocrea S, Constantino G, Mazzon E et al. Beneficial effects of melatonin in a rat model of splanchnic artery occlusion and reperfusion. J Pineal Res. 2000; 28(1): 52.

12. Sewerynek E, Reiter RJ, Melchiorri D et al. Oxidative damage in the liver induced by ischemia reperfusion: protection by melatonin. Hepatogastroenterology. 1996; 43(10): 898 .

13. Vural H, Sabuncu T, Aíslan SO et al. Melatonin inhibits lipid peroxidation and stimulates the antioxidant status of diabetic rats. J Pineal Res. 2001; 31(3): 193.

14. Reiter RJ, Tan DX, Qi W et al. Pharmacology and phisiology of melatonin in the reduction of oxidative stress in vivo. Biol Signals Recept. 2000; 9(3-4): 160.

15. Karaçal N, Ambarcio lu O, Topal $U$ et al. Enhancement of dorsal random-pattern skin flap survival in rats with topical lidocaine and prilocaine (EMLA): enhancement of flap survival by EMLA. J Surg Res. 2005; 124(1): 134.

16. Covino BG. Pharmacology of local anaesthetics agents. Br J Anaesth. 1986; 58: 701.

17. Petry JJ, Wortham KA. The anatomy of the epigastric flap in the experimental rat. Plast Reconstr Surg. 1984; 74(3): 410.

18. Dunn RM, Mancoll J. Flap modelsin the rat: a review and reappraisal. Plast Reconstr Surg. 1993; 91(7): 1374.

19. Gurlek A, Celik M, Parlakpinar $\mathbf{H}$ et al. The protective effect of melatonin on ischemia-reperfusion injury in the groin (inferior epigastric) flap model in rats. $J$ Pineal Res. 2006; 40(4): 312.

20. Eskitascioglu T, Karaci S, Canoz $\mathbf{O}$ et al. The Impact of Lidocaine on Flap Survival Following Reperfusion Injury. J Surg Res. 2009; 167(2): 323. 